\title{
Coding and Modulation Techniques for High Spectral Efficiency Transmission in 5G and Satcom
}

\author{
Haesik Kim \\ VTT Technical Research Centre of Finland, \\ Kaitoväylä 1, FI-90571, Oulu, Finland \\ haesik.kim@vtt.fi \\ (Invited paper)
}

\begin{abstract}
Achieving high spectral efficiency is the key requirement of 5G and Satcom systems because it provides us with much lower cost per bit. In order to achieve high spectral efficiency, channel coding and modulation are the key part of the physical layer. Basically, high spectral efficiency can be achieved when adopting a high order modulation and low code rate at a high SNR. However, the transmit power is limited in practical wireless communication systems. The high order modulation and low code rate is restrictively used. Thus, the integrated version of 5G and Satcom needs a new type of channel coding scheme. In this paper, we look into $5 \mathrm{G}$ requirements and Satcom's role in 5G, review candidate error correction coding schemes for $5 G$ and future Satcom in terms of spectral efficiency, and evaluate the performance of the candidate error correction codes.
\end{abstract}

Index Terms-Spectral efficiency, Error correction codes, Turbo codes, LDPC codes, 5G, Satcom, etc.

\section{INTRODUCTION}

$\mathrm{W}$ E face a strong demand to deliver higher data rates in various wireless communication systems, which is driven by the growth in the video traffic and new applications. In the $5^{\text {th }}$ generation $(5 \mathrm{G})$ systems, an integrated system of cellular networks supporting a high data rate and satellite communication systems covering a large area is envisioned. In order to achieve higher data rates, we should consider many design parameters such as bandwidth, energy consumption, latency and complexity. Sometimes they become constraint condition. We should hurdle those barriers and achieve $5 \mathrm{G}$ system goals.

Cellular communication systems have evolved from $1 \mathrm{G}$ to $5 \mathrm{G}$ and provided us with different services. For example, $1 \mathrm{G}$ focused on voice services, $2 \mathrm{G}$ improved voice services and supported text messaging services, $3 \mathrm{G}$ integrated voice services and affordable mobile internet services, 4G supports high capacity mobile multimedia service and $5 \mathrm{G}$ demands higher capacity, lower latency and more consistent experience. In terms of error correction coding techniques, there was a big jump from $2 \mathrm{G}$ to $3 \mathrm{G}$ because Turbo codes [1] and Low
Density Parity Check (LDPC) codes [2, 3] are included. It was paradigm change. However, error correction coding schemes in $3 \mathrm{G}$ and $4 \mathrm{G}$ are almost same. Another big jump from $4 \mathrm{G}$ to $5 \mathrm{G}$ is expected because $5 \mathrm{G}$ faces news challenges such as much higher spectral efficiency and severe interferences in heterogeneous networks. In 2008, Digital Video Broadcasting - Cable 2 (DVB-C2) standard [4] was announced. It offers 16QAM to 4096QAM modulation with code rates of $1 / 2$ to $9 / 10$ for spectral efficiency of $10.8 \mathrm{bits} / \mathrm{s} / \mathrm{Hz}$. However, it is hard to achieve under real operation conditions such spectral efficiencies with acceptable effort. Basically, the transmission rate increases will be achieved by spectral efficiency increases (ex. using a high order modulation). However, a high order modulation scheme would degrade system performance because it is vulnerable to wireless channel impairments of a practical wireless communication system. The current cellular systems and satellite communication systems include LDPC codes and Turbo codes. These techniques are provably suboptimal solution in terms of information theory and they are not optimal solution when combining with a high order modulation technique. Thus, an alternative approach should be investigated. It is essential to develop a new error correction coding technique for $5 \mathrm{G}$ and Satellite communication (Satcom) systems.

Spectral efficiency $\eta$ represents the information rate over a given bandwidth and can be defined as follows:

$$
\eta=\frac{R}{W}[\mathrm{bits} / \mathrm{s} / \mathrm{Hz}]
$$

when one coding scheme transmits $R$ [bits/s] over an AWGN of bandwidth $W[\mathrm{~Hz}]$. Shannon described the relationship between the spectral efficiency and signal to noise ratio (SNR) for the reliable transmission as follows:

$$
\eta<\log _{2}(1+\mathrm{SNR})
$$

As we can observe (2), the spectral efficiency is increased depending on SNR. Basically, high spectral efficiency can be achieved when adopting a high order modulation and low code 
rate at a high SNR. However, the transmit power is limited in practical wireless communication systems as follows:

$$
\mathrm{SNR}>2^{\eta}-1
$$

A low code rate provides us with a low bit error rate (BER) but redundancy is increased. Thus, a high order modulation scheme and a low code rate can be used only when channel condition is good.

In many cellular systems, the spectral efficiency is adopted in order to evaluate how efficiently the bandwidth is utilized. The spectral efficiency of cellular systems ( $1 \mathrm{G}$ to $4 \mathrm{G}$ ) has been increased as shown in table 1 .

Table 1. Spectral efficiency of cellular systems.

\begin{tabular}{|c|c|c|c|c|}
\hline & 1G (AMPS) & $\begin{array}{c}2 \mathrm{G} \\
(\mathrm{GSM})\end{array}$ & $\begin{array}{c}3 \mathrm{G} \\
\text { (CDMA200 } \\
\text { 0 EV-DO } \\
\text { Rev.A) }\end{array}$ & $\begin{array}{c}4 \mathrm{G} \\
\text { (LTE) }\end{array}$ \\
\hline \hline $\begin{array}{c}\text { Max link } \\
\text { spectral } \\
\text { efficiency } \\
\text { [bits/s/Hz] }\end{array}$ & 0.001 & 0.52 & 2.5 & $\begin{array}{c}4.08 \\
(\mathrm{SISO}) / \\
16.32(4 \times 4 \\
\text { MIMO) }\end{array}$ \\
\hline
\end{tabular}

As we can see in table 1 , spectral efficiencies from $1 \mathrm{G}$ to $4 \mathrm{G}$ have been significantly improved. Especially, there was a big jump from $1 \mathrm{G}$ to $2 \mathrm{G}$. The role of coding and modulation techniques was significant. In addition, Multiple Input and Multiple Output (MIMO) techniques are key techniques in 4G systems. The importance of MIMO techniques will be emphasized in $5 \mathrm{G}$ systems. In order to achieve high spectral efficiency (Beyond $5 \mathrm{bits} / \mathrm{s} / \mathrm{Hz}$ ), the integrated version of $5 \mathrm{G}$ system and Satcom needs a new type of channel coding schemes. In this paper, we review candidate error correction coding schemes for $5 \mathrm{G}$ and Satcom systems and evaluate their performance.

The remaining parts of this paper are organized as follows: In section II, we look into 5G standardization plan and requirements of industry and academia and take a look at Satcom's role in $5 \mathrm{G}$ systems. In section III, candidate channel coding schemes for $5 \mathrm{G}$ and Satcom systems are introduced. In section IV, their performances are evaluated. Finally, section $\mathrm{V}$ contains the conclusion.

\section{5G SYSTEM AND SATCOM’S ROLE}

In this section, we discuss $5 \mathrm{G}$ system standardization and requirements and then look into the role of Satcom in $5 \mathrm{G}$ systems.

\section{A. $5 G$ Standardization.}

The $5 \mathrm{G}$ standardization and regulation will be developed as follows: Firstly, the international regulator such as the International Telecommunication Union (ITU) defines the spectrum allocation for $5 \mathrm{G}$ services. The regional regulatory bodies such as European Conference of Postal and
Telecommunications Administrations (CEPT) in Europe and Federal Communications Commission (FCC) in US play quite important role in the decision making process in the ITU. They build a regional consensus even if there are national representatives in ITU meetings. Secondly, after spectrum allocation for a certain service, the ITU may label certain allocations for a certain technology and determine the requirements for the technology. For the requirements some standardization bodies such as the 3rd Generation Partnership Project (3GPP) and the Institute of Electrical and Electronics Engineers (IEEE) make their contributions to the ITU, where they guarantee that their technology will meet the requirements. The actual technology is specified in the internal standardization such as 3GPP and IEEE. Some standards are established in the regional level. The regulators are responsible for spectrum licensing and protection.

The ITU's Radio communication Sector (ITU-R) Working Party 5D has the leading role and plans to deploy the $5 \mathrm{G}$ system in 2020 [5]. The 3GPP also plans to enhance LTE system and meet the $5 \mathrm{G}$ requirements of ITU [6]. The 5G target of the 3GPP is to (1) improve LTE capacity and performance and (2) address a new business segment. The $5 \mathrm{G}$ timeline of ITU and 3GPP is summarized in figure 1.

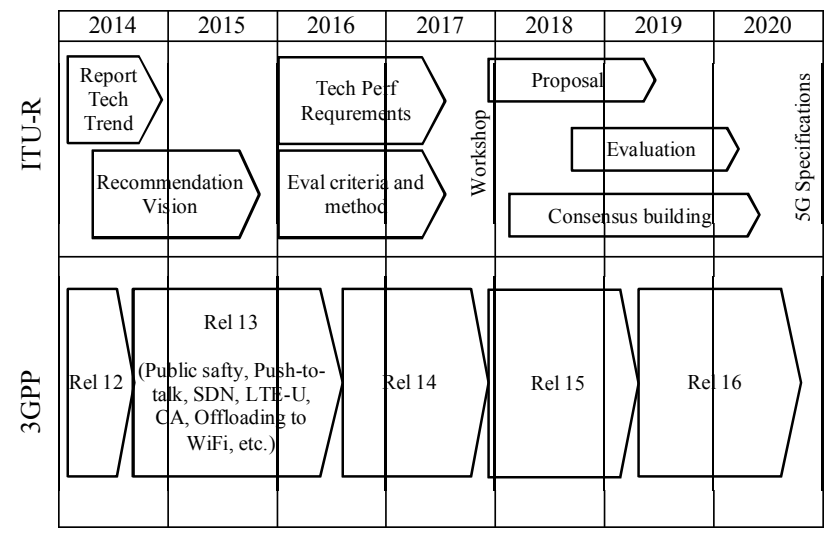

Fig. 1. 5G timeline of ITU-R and 3GPP.

\section{B. $5 G$ Requirements}

In [7], 5G system requirements are presented. The data rate needs to increase by roughly 1000 times from $4 \mathrm{G}$ to $5 \mathrm{G}$. The data traffic needs to increase by 10000 times. The $5 \mathrm{G}$ edge rate (or 5\% rate) ranges from $100 \mathrm{Mbps}$ to as much as $1 \mathrm{Gbps}$. The $5 \mathrm{G}$ needs to support a roundtrip latency of about $1 \mathrm{~ms}$. More than 10 to 100 times higher number of connected devices are anticipated in 5G system. The energy efficiency (Joules per bit) needs to fall by at least 100 times. In addition, accurate positioning of the devices, strong level of security, smooth mobility between different types of radio access networks, and flexible and very dense deployments are required. There are other views of $5 \mathrm{G}$ requirements from industry and academia as shown in table 2 . 
Table $2.5 \mathrm{G}$ requirements of industry and academia.

\begin{tabular}{|c|c|c|c|c|c|}
\hline & $\begin{array}{c}\text { Samsung } \\
{[8]}\end{array}$ & Nokia [9] & $\begin{array}{c}\text { NTT } \\
\text { Docomo } \\
{[10]}\end{array}$ & Metis [11] & $\begin{array}{c}\text { Academia } \\
{[7]}\end{array}$ \\
\hline \hline Data rate & $50 \mathrm{x}$ & $10 \mathrm{x}$ & $100 \mathrm{x}$ & $100 \mathrm{x}$ & $1000 \mathrm{x}$ \\
\hline $\begin{array}{c}\text { Cell edge } \\
\text { data rate }\end{array}$ & $>1 \mathrm{Gbps}$ & $100 \mathrm{Mbps}$ & - & - & $>1 \mathrm{Gbps}$ \\
\hline Latency & $<1 \mathrm{~ms}$ & $<1 \mathrm{~ms}$ & $<1 \mathrm{~ms}$ & $<5 \mathrm{x}$ & $<1 \mathrm{~ms}$ \\
\hline $\begin{array}{c}\text { Connected } \\
\text { devices }\end{array}$ & $10 \mathrm{x}$ & $100 \mathrm{x}$ & $100 \mathrm{x}$ & $100 \mathrm{x}$ & $100 \mathrm{x}$ \\
\hline $\begin{array}{c}\text { Energy } \\
\text { efficiency }\end{array}$ & - & $\begin{array}{c}10 \mathrm{years} \\
\text { M2M }\end{array}$ & - & $\begin{array}{c}10 \mathrm{x} \text { longer } \\
\text { battery life }\end{array}$ & $<100 \mathrm{x}$ \\
\hline $\begin{array}{c}\text { Cost } \\
\text { efficiency }\end{array}$ & $10 \mathrm{x}$ & - & - & - & \\
\hline \begin{tabular}{c} 
Traffic \\
\hline
\end{tabular} & - & $10000 \mathrm{x}$ & - & $>1000 \mathrm{x}$ & \\
\hline
\end{tabular}

As we can observe in table 2, academia sets more aggressive targets and industry sets more realistic targets. However, their gap is not so big. They approximately achieve consensus on $5 \mathrm{G}$ requirements.

Among those 5G requirements, achieving high spectral efficiency is the key requirement because it provides us with much lower cost per bit. In order to achieve high spectral efficiency in 5G system, channel coding and modulation are they key part of the $5 \mathrm{G}$ physical layer. The channel coding and modulation of the $5 \mathrm{G}$ system requires to achieve better spectral efficiency, support multi-terminal encoding and decoding schemes in cooperative communications or heterogeneous networks and reduce transmission and computation power.

\section{Satcom's role in $5 G$ systems.}

The 5G system targets to integration of access technologies into one seamless experience. One important trend is integration and interoperability of terrestrial communication systems and satellite communication systems [12]. Satom provides us with good services at specific area (sea, artic area, dessert, mountain, etc.) terrestrial communication systems do not cover. However, it does not compete well at terrestrial communication service area and focuses on specific area it shows off its merits such as wide range coverage, instant infrastructure and scalability. In this point of view, satellite $\mathrm{TV}$ and digital broadcasting have been a big success.

The integration of two systems is now actively investigated. For example, Digital Video Broadcasting - Satellite services to Handhelds (DVB-SH) as an integrated/hybrid satellite and terrestrial system is defined in [13]. Figure 2 illustrates conceptual network of integrated satellite and terrestrial system.

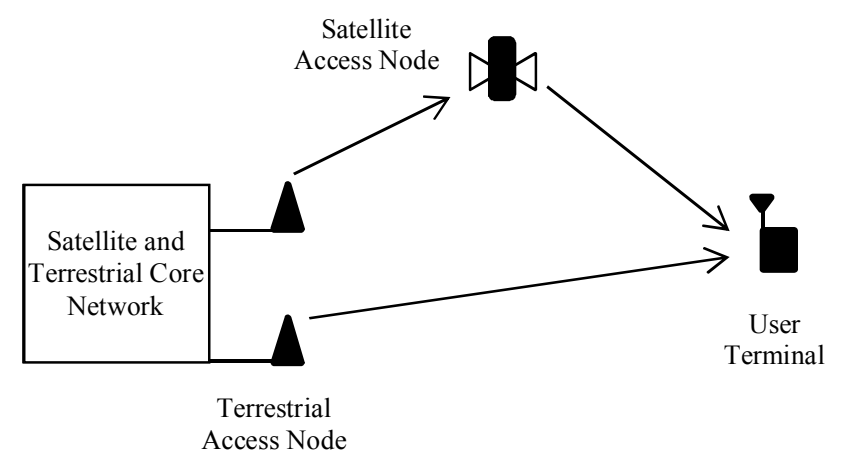

Fig. 2. Integrated satellite and terrestrial system.

As we can see figure 2, the user terminal can receive a service from both satellite access node and terrestrial access node depending on environments. For example, terrestrial access node mainly covers users in urban area, the users in suburban area receive the signal from both satellite and terrestrial access nodes, and the users in rural area (or specific area terrestrial access nodes cannot cover.) are covered by satellite access node. When both access nodes are operated and they use the same frequency bands, MIMO/Cooperative communication techniques can be adopted in order to improve link quality. In [14], the key contributions of Satcom in 5G systems are described as follows: (1) coverage extension of $5 \mathrm{G}$ networks, (2) multimedia service improvement to the edge user, (3) terrestrial network off-loading using satellite backhaul and traffic control via satellite and (4) network resilience by integrating satellite with terrestrial. These contributions utilize the merits of Satcom systems. It will help us to meet $5 \mathrm{G}$ requirements. Especially, if we allocate satellite resources to cell edge users, we can improve cell edge data rate improvement and optimize the infrastructure dimensioning. In addition, a high speed broadband satellite service enables us to extend reliable $5 \mathrm{G}$ services to high mobility users.

\section{Coding And Modulation for High Spectral EFFICIENCY}

In this section, candidate channel coding schemes are investigated for $5 \mathrm{G}$ and Satcom systems. Especially, a family of Turbo codes and LDPC codes is focused.

\section{A. Turbo Codes and Duo-binary Turbo Codes}

The turbo codes achieved very low error probability which is close to the Shannon limit. The important design criteria of the turbo codes are to find suitable component codes which maximize the effective free distance [15] and to optimize the weight distribution of the codewords at a low $\mathrm{E}_{\mathrm{b}} \mathrm{N}_{0}$ [16].

One disadvantage of the original turbo codes is that error floor occurs at BER $10^{-5}$ due to poor minimum Hamming distance. possible solutions are to (1) design a good interleaver, (2) increase the number of memories and (2) use the non-binary turbo codes such as duo binary turbo codes or 3D turbo codes. In this section, we takes a look at duo-binary turbo codes [17] as one candidate 5G and Satcom channel coding. Duo-binary turbo codes are composed of two Circular 
Recursive Systematic Constituent (CRSC) codes with two inputs. Its information bits are encoded pair wise. CRSC encoders do not need any tail bits.

In [18], many advantages of duo-binary turbo codes are described as follows: (1) better convergence, (2) larger minimum distances, (3) less sensitivity to puncturing patterns, (4) reduced latency and (5) robustness of the decoder. Especially, the decoder of duo-binary turbo codes can be implemented much simply because the gap between MAP algorithm and its simplified version as component decoders is very small. Above all things, it is very well matched with a high order modulation [19]. They are already adopted into several standards as shown in table 3 .

Table 3. Turbo codes and duo-binary turbo codes in standards.

\begin{tabular}{|c|c|c|c|c|}
\hline Standard & $\begin{array}{c}\text { Type of } \\
\text { Turbo Codes }\end{array}$ & Code Rates & Polynomials & Termination \\
\hline \hline $\begin{array}{c}3 \mathrm{G} \\
\text { (UMTS and } \\
\text { cdma2000) }\end{array}$ & Turbo codes & $\begin{array}{c}1 / 2,1 / 3 \text { and } \\
1 / 4\end{array}$ & $13,15,17$ & Tail bits \\
\hline $\begin{array}{c}4 \mathrm{G} \\
\text { (WiMAX) }\end{array}$ & $\begin{array}{c}\text { Duo-binary } \\
\text { Turbo codes }\end{array}$ & $1 / 2 \sim 7 / 8$ & 13,15 & Circular \\
\hline DVB-RCS & $\begin{array}{c}\text { Duo-binary } \\
\text { Turbo code }\end{array}$ & $1 / 3 \sim 6 / 7$ & 13,15 & Circular \\
\hline CCSDS & Turbo codes & $\begin{array}{c}1 / 2,1 / 3,1 / 4 \\
\text { and } 1 / 6\end{array}$ & $23,33,25,37$ & Tail bits \\
\hline
\end{tabular}

\section{B. $L D P C$ and Non-binary $L D P C$}

In 1962, R. Gallager [20] originally invented LDPC codes in his $\mathrm{PhD}$ thesis. However, the LDPC codes didn't get attention until D.J.C Mackay and R.M. Neal [21] rediscovered because the era of transistors is just started and the hardware technology does not cover the complexity of LDPC encoding and decoding at that time. After the turbo codes are emerged in 1993, many researchers make an effort to understand how the turbo codes achieve near Shannon limit and try to find another new error correction code. In 1996, Mackay and Neal designed a new linear block code including many similar features of turbo codes such as randomness, large block length and iterative decoding. They soon realised that the new codes are almost same as LDPC codes by Gallager. After that, irregular LDPC codes [22] as generalization of Gallager's LDPC codes are introduced by Luby in 1998. The irregular LDPC codes became the most powerful error control codes as of now. When comparing with the turbo codes, LDPC codes have several advantages. Firstly a random interleaver is not required. Secondly, it has a better block error rate and a lower error floor. Thirdly, iterative decoding of LDPC codes is simpler operation even if it requires more iterations. The most highlighted advantage is that it is patent-free.

Non-binary LDPC codes [23] can include nonzero elements of $\boldsymbol{H}$ matrix like Reed-Solomon (RS) codes. Non-binary LDPC codes using iterative Belief Propagation (BP) show us good performance at medium code lengths $(500 \leq N \leq 3000)$ and high order modulation communications (greater than
16QAM). However, the decoding complexity remains a major obstacle for their commercial applications.

\section{Other Candidate Channel Codes}

In this section, we briefly take a look at two other candidate channel codes: Sparse Regression Codes and Polar Codes. Sparse Regression Codes [24] as a new class of codes for Gaussian multi-terminal source and channel coding are designed using the statistical framework of high-dimensional linear regression. They are rate-optimal codes with low complexity for compression and transmission. They are suitable for cooperative communications.

Polar codes [25] are first constructive coding to provably achieve channel capacity using Kronecker product. Its characteristic can be summarized as follows: (1) the block length of a polar code is normally a power of two but the code length can be adjusted by usual code shortening methods, (2) the rate of a polar code can be adjusted to any number between 0 and 1 in increments of $1 / N$, (3) polar codes are available in systematic form. (4) polar codes have a recursive structure which makes them suitable for low-complexity implementations and (5) polar codes have no error floor.

\section{Simulation}

In this section, the performances of duo-binary turbo codes and non-binary LDPC codes are investigated. In the first simulation, the frame error rate (FER) performance of duobinary turbo code is investigated. Simulation configuration is as follows: 8-state duo-binary convolutional turbo codes, Max-log-MAP decoding, frame size 8000, 10 iterations and AWGN.

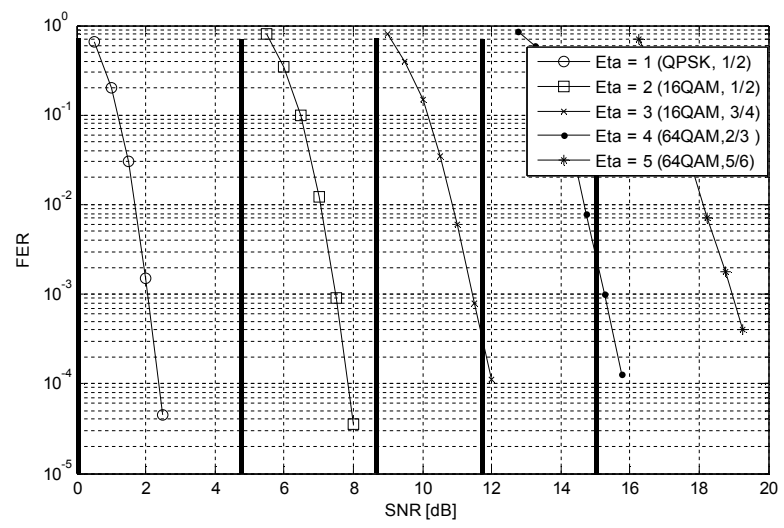

Fig. 3. Required SNR to achieve the spectral efficiency.

In figure 3, spectral efficiencies $(\eta=1,2,3,4$ and 5) are achieved by different modulation schemes and code rates ((QPSK, 1/2) , (16QAM, 1/2), (16QAM, 3/4), (64QAM, 2/3), and (64QAM, 5/6)), respectively. Each vertical line represents the minimum required SNR to achieve each spectral efficiency. As we can observe the figure, the minimum required SNR is very low. In addition, practical wireless communication systems include many other factors (synchronization overhead, frequency and phase offset, etc.) which degrade the performance. Thus, it is very challenging to 
meet high spectral efficiency (Beyond $5 \mathrm{bits} / \mathrm{s} / \mathrm{Hz}$ ) in practice. It has been shown in [26] that the spectral efficiency of the regular $(2,4)$ GF $\left(2^{q}\right)$ LDPC code increases with the field order $q$, code length and modulation order. In the second simulation, spectral efficiency of non-binary LDPC code is investigated. Simulation configuration is set as follows: regular $(2,4)$ LDPC over $\mathrm{GF}(256)$, target BER $10^{-5}$, gray labelling, belief propagation decoding, frame size $\mathrm{N}=2000$ and 40000, and AWGN.

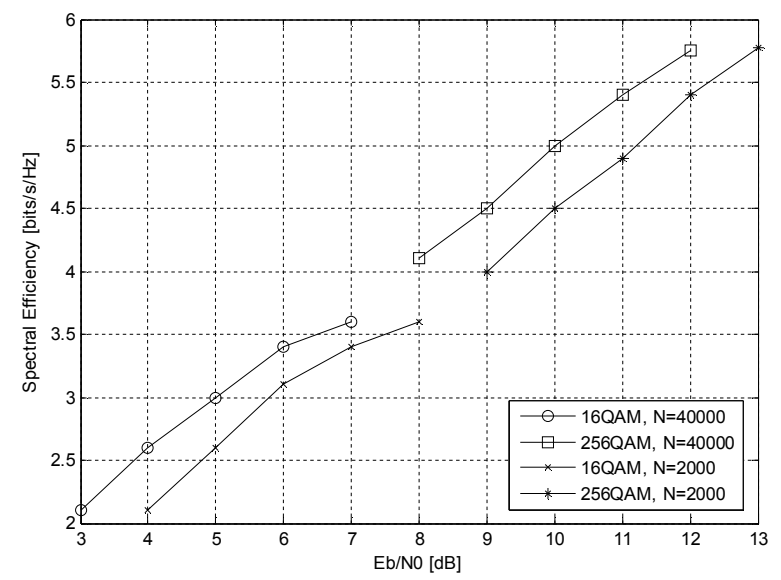

Fig. 4. Non-binary $(2,4)$ LDPC codes over GF(256).

As we can observe in figure 4, spectral efficiency increases according to modulation order and frame size. Non-binary LDPC code with the large codeword lengths achieved high spectral efficiency (Beyond $5 \mathrm{bits} / \mathrm{s} / \mathrm{Hz}$ ) at relatively low power. This confirms that results obtained in [26].

From two simulations, we realized duo-binary turbo codes and non-binary turbo codes can achieve high spectral efficiency at relatively low power. However, the required transmit power of $5 \mathrm{G}$ is much lower than we expect. Thus, further improvements of both error correction coding schemes are needed.

\section{CONCLUSION}

High spectral efficiency (Beyond 5 bits $/ \mathrm{s} / \mathrm{Hz}$ ) is one of key aspects in 5G and Satcom systems. When we design 5G and Satcom systems, there are several constraints such as bandwidth, energy consumption, latency and complexity. We should hurdle those barriers and achieve the goal. Error correction codes as one key technique of $5 \mathrm{G}$ and Satcom will be able to improve spectral efficiency of $5 \mathrm{G}$ and Satcom systems. Error correction codes of $5 \mathrm{G}$ systems requires to achieve a better spectral efficiency, support multi-terminal encoding and decoding schemes in heterogeneous networks, and reduce transmission and computation power. The integrated system of $5 \mathrm{G}$ and Satcom should find a new type of channel coding scheme. In this paper, we reviewed several candidate error correction codes for $5 \mathrm{G}$ and Satcom systems and evaluated their performances in terms of spectral efficiency.

\section{ACKNOWLEDGEMENT}

This work was done in the 5GTN (decision number 3656/31/2014) funded by Tekes (the Finnish Funding Agency for Innovation).

\section{REFERENCES}

[1] C. Berrou, A. Glavieux and P. Thitimajshima, "Near Shannon limit error-correcting coding and decoding: Turbo-codes," Proc. Of ICC '93, Geneva, pp.1064-1070, 23-26 May 1993.

[2] R. G. Gallager, Low-Density Parity-Check Codes, Cambridge, MA: MIT Press, 1963

[3] D. J. C. MacKay and R. M. Neal, "Near Shannon limit performance of low density parity check codes," Electron. Lett., vol. 32, pp. 1645-1646, Aug. 1996.

[4] Digital Video Broadcasting (DVB); Frame structure channel coding and modulation for a second generation digital transmission system for cable systems (DVB-C2), DVB Document A138, June 2010

[5] http://www.itu.int/en/ITU-R/study-groups/rsg5/rwp5d/imt2020/Pages/default.aspx

[6] http://www.3gpp.org/news-events/3gpp-news/1614-sa_5g

[7] J.G.Andrews, S. Buzzi, W. Choi, S.V. Hanly, A. Lozano, A.C.K. Soong, and J.C. Zhang, "What Will 5G Be?" IEEE Journal on Selected Areas in Communications, vol. 32, Issue:6, pp.1065-1082, June 2014.

[8] Samsung Electronics DMC R\&D Center, "5G Vision," Feb. 2015

[9] Nokia, "5G use cases and requirements," networks.nokia.com

[10] NTT Docomo, "5G: Next-Generation Mobile Communications System," 2014

[11] Metis Deliverable D1.1, "Scenarios, requirements and KPIs for 5G mobile and wireless system," Document Number: ICT-317669METIS/D1.1.

[12] B. Evans, M. Werner, E. Lutz, M. Bousquet, G.E. Corazza, G. Maral, R. Rumeau and E. Ferro, "Integration of satellite and terrestrial systems in future multimedia communications," IEEE Wireless Communications, vol. 12 , issue:5, Oct. 2005.

[13] Annex 12 to ITU-R Working Party 4B Chairman's Report on the Twenty Sixth Meeting. Document 4B/85, 27 May 2009.

[14] NetWorld2020's - SatCom WG The role of satellites in 5G, July 2014.

[15] D. Divslar and R.J. McEliece, "Effective Freee Distance of Turbo Codes", Electronic Letters, vol. 32, no. 5, pp. 445-446, 29th Feb 1996.

[16] D. Divsalar and F. Pollara, "One the Design of Turbo Codes," TDA Progress Report 42-123, Jet Propulsion Lab, Pasadena, California, pp.99-121, 15th November 1995.

[17] C. Douillard and C. Berrou, "Turbo Codes with rate-m/(m+1) constituent convolutional codes," IEEE Transactions On Communications, vol. 53, pp.1630-1638, Oct 2005.

[18] C. Berrou, M. Jézéquel, C. Douillard, S. Kerouédan and L. Conde Canencia, "Duo-binary turbo codes associated with high-order modulations," 2nd ESA Workshop on Tracking Telemetry and Command Systems for Space Applications, TTC'2001, Noordwijk, the Netherlands, Oct. 2001

[19] S.L. Goff, A.Glavieux and C. Berrou, "Turbo-codes and high spectral efficiency modulation," Proc. of IEEE International Conference on Communications (ICC) 1994, pp. 645-649, May1994.

[20] R. G. Gallager, "Low Density Parity Check Codes," Monograph, M.I.T. Press, 1963.

[21] D.J.C. MacKay and R. M. Neal, "Near Shannon Limit Performance of Low Density Parity Check Codes," Electronics Letters, July 1996.

[22] M.G. Luby, M. Mitzenmacher, M.A.Shokrollahi, and D.A. Speilman, "Improved low-density parity check codes using irregular graphs and belief propagation," IEEE Int. Symp. on Information Theory (ISIT), 1621 Aug, 1998.

[23] M. C. Davey and D. Mackay, "Low-density parity check codes over GF(q)," IEEE Comm. Letters, vol. 2, pp. 165-167, June 1998.

[24] A. Barron and A. Joseph, "Toward fast reliable communication at rates near capacity with Gaussian noise," Proc. of IEEE ISIT 2010.

[25] E. Arikan, "Channel polarization: A method for constructing capacity achieving codes for symmetric binary-input memoryless channels," IEEE Trans. Inf. Theory, vol. 55, pp. 3051 -3073, July 2009.

[26] D. Declercq, M. Cloas and G. Gelle, "Regular GF $\left(2^{\mathrm{q}}\right)$-LDPC modulations for higher order QAM-AWGN channels," Proc. Of International Symposium on Information Theory and its Applications (ISITA) 2004, Parma, Italy, 10-13 Oct. 2004. 